Rapid response

\section{International Journal of \\ Molecular Medicine}

Editor and publisher Demetrios A.

Spandidos

12/yr. Europe $\$ 600$ (institutional), $\$ 300$

(personal); elsewhere $\$ 650$ (institutional),

$\$ 350$ (personal)

\section{Jeffrey M.Leiden}

Basic scientists, physicians and venture capitalists all agree that twenty-first century medicine will be based increasingly on our understanding of the molecular genetic causes of human disease. The scheduled completion of the human genome project in 2005, along with advances in human genetics, immunology, molecular pathogenesis, structural biology and gene therapy are creating an interdisciplinary field of molecular medicine that promises to revolutionize the ways that we investigate, diagnose, and treat many acquired and inherited diseases.

We are already beginning to identify the genes involved in prevalent diseases such as hypertension, diabetes and cancer. The discovery of these genes and their functions is providing important pathophysiologic insights, and molecular probes can accurately assess a patient's susceptibility for disease long before they become symptomatic. Of equal importance, access to these genes and their protein products provides new targets for rational drug design, drug screening and somatic gene therapy.

This revolution in molecular medicine has created a vacuum in the biomedical literature. And, as is usually the case, medical publishers have rushed in to fill the void. New molecular medicine journals, including Nature Medicine, Molecular Medicine, Human Molecular Genetics and Molecular Medicine Today, publish original research articles and reviews in this area. Meanwhile, established journals such as the Journal of Clinical Investigation are increasingly filled with molecular medicine manuscripts. Given this cornucopia of literature, one could justifiably ask if we need yet another journal on molecular medicine. Only time will tell, but a review of the first five issues of the International Journal of Molecular Medicinesuggests that the answer may be yes.

This journal's goal is the rapid publication of original work and reviews concerning the molecular mechanisms of human disease. Of necessity, this is a wide purview, encompassing the fields of human genetics, immunology, virology, pharmacology, pathology and gene therapy among others. Manuscripts can be submitted directly for peer review or can be contributed by members of the editorial board, who are experts in fields related to molecular medicine. The journal includes basic papers dealing with the molecular pathways involved in pathological cell function, and more clinical papers describing novel pharmacologic and genetic therapies. Of equal importance, each issue contains three to five review articles that summarize a variety of interesting topics at the interface of molecular biology and clinical medicine. In general the articles are well written and the illustrations and graphics are first rate.

Compared with many other journals on the subject, the time from submission to acceptance is remarkably short, averaging less than one month in the issues under review. One of the most interesting aspects is the preponderance of articles submitted from outside the United States. This appears to reflect the international flavour of the editorial board and the increasing interest in molecular medicine in Europe and Asia. Some articles, however, could benefit from more careful grammatical editing. Jeffrey M. Leiden is in the Chief Section of Cardiology, University of Chicago, 5841 South Maryland Avenue, Chicago, Illinois 60637, USA.

\section{A fertile and dynamic sea}

Current Opinion in Chemical Biology

Editors Donald Hilvert and Steven Ley Current Biology. 6/yr. Print, £125, \$220

(personal); $£ 60, \$ 120$ (student). On-line

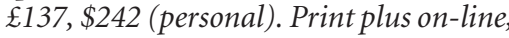

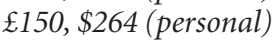

\section{Brent L. Iverson}

Chemical tools are increasingly being applied to, and chemists are increasingly being inspired by, the molecules of living systems as well as the living systems themselves. As scientists attempt to ride these rapidly advancing currents of progress, new paradigms quickly arise and become part of the fertile and dynamic sea of ideas and experimental results that make up chemical biology.

Current Opinion in Chemical Biology, first published in June 1997, covers timely and important topics of bioorganic as well as bioinorganic chemistry, along with relevant advances in enzymology and medicinal chemistry. Editors Donald Hilvert and Steven Ley have enlisted an impressive group of world leaders in the field to oversee the writing of short reviews by experts on recent developments in the most active areas of chemical biology research. Journal issues this year are devoted to interaction, assembly and processing (February); biocatalysis and biotransformation (February); bioinorganic chemistry (April); combinatorial chemistry (June); next generation therapeutics (August); analytical techniques (October); mechanisms (October); model systems (December); and biopolymers (December). The review articles are of the highest scientific calibre and are focused on recent literature as viewed through the eyes of those who know and understand that literature best. The reviews are generally eight to ten pages in length, long enough to explore thoroughly all essential ideas, but not so long that the reader is set adrift in details.

Current Opinion in Chemical Biology also has 'paper alert' and 'web alert' sections to help readers negotiate the large number of new currents of chemical biology literature, both published and electronic. In these 'alert' sections, scientists representing the journal's key topic areas highlight important recent papers or outstanding websites, along with a concise summary of key results and their significance.

To ride new waves in chemical biology, scientists must know how recent scientific advances are moving around them. A subscription to Current Opinion in Chemical Biology will provide excellent guidance to scientists on how best to harness the onrush of new advances and trends, thereby preventing a scientific 'wipe out' caused by being overtaken from an important new direction by something they did not see coming. In scientific research, no good-looking lifeguard comes to the rescue.

Brent L. Iverson is in the Department of Chemistry and Biochemistry, University of Texas at Austin, Austin, Texas 78712, USA

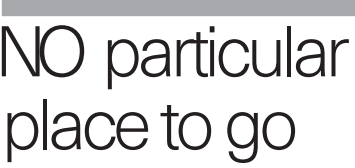

Nitric Oxide: Biology and Chemistry Editor-in-chief Louis J. Ignarro

Academic Press. 6/yr. North America \$220, elsewhere \$262 (institutional); North

America \$110, elsewhere \$132 (personal)

\section{John Garthwaite}

There is no doubting that the identification of nitric oxide (NO) as a biological signalling molecule has had an extraordinary impact. It all started about ten years ago when

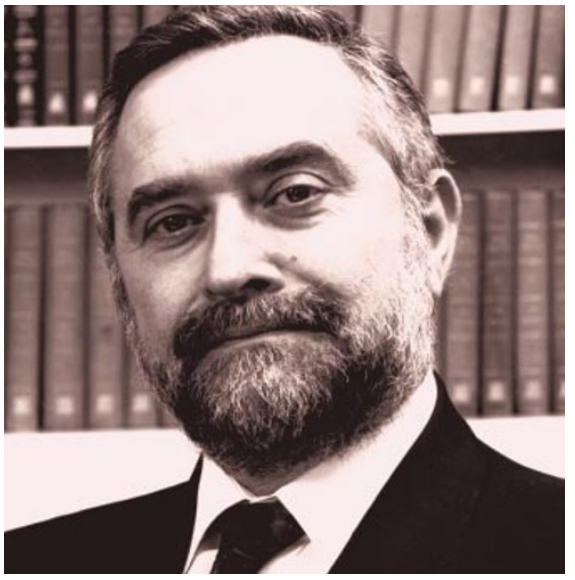

Salvador Moncada: a pioneer in NO research. 
researchers in three previously disparate areas of research (vascular biology, neuroscience and immunology) all stumbled on the same molecule.

Within a short period of time, it was realized that $\mathrm{NO}$ was involved in the regulation of blood vessels, in communication in the brain, and in immunological defence against invading organisms. Now, NO impinges on almost all areas of biology. Not only is it important physiologically as a messenger that conveys signals from one cell to another in many different tissues but, through its toxic effects, it is also a likely player in many pathological processes.

So it was decided that we need to have a journal devoted specifically to the molecule, Nitric Oxide: Biology and Chemistry. But do we? It could also be said that, because of its importance to so many areas of biology, a journal solely concerned with NO is a nonstarter: NO is really only interesting in context. After all, we don't have, or need, journals specifically about glutamate or acetylcholine or inositol trisphosphate.

Viewed from this perspective, there is no shortage of journals in which to publish and read about NO research. In fact, almost any one will do.

Nitric Oxide: Biology and Chemistry is a spin-off from Archives of Biochemistry and Biophysics and is also referred to as 'Part B' of that journal. The first issue appeared in February 1997 and, so far, only eight issues have been published (six of which were from last year) so it is possibly a little early to expect the journal to have found its flavour.

The editors would like it to be a repository of papers about all aspects of the biology and chemistry of NO, but this is probably unrealistic. To date, there have been a few reviews, and a modest number (up to ten) of research papers per issue (except the latest: volume two, number two, which is a book of abstracts). Some of the better papers have come from the editorial advisory board's research groups. These aside, the journal appears to be falling into its default guise, which is Archives of Biochemistry and Biophysics Part B. The parent journal has long had a penchant for chemically oriented research into reactive free radicals, and $\mathrm{NO}$ does have this chemical characteristic (although it is not very reactive).

Of course it is important that the chemistry of NO and related species, such as the much more reactive peroxynitrite anion, does become better understood. Indeed, it is just this type of research that one imagines could find a home in a journal devoted exclusively to NO. The problem is that, when the chemistry is not constrained within a biological framework, you can get free radicals to do almost anything.

John Garthwaite is at the Wolfson Institute for

Biomedical Research, University College London, 140 Tottenham Court Road, London W1P 9LN, UK.

\section{Venturing outside the laboratory}

\section{Field Analytical Chemistry and Technology \\ Editor Henk L. C. Meuzelaar \\ Wiley. 6/yr. USA \$195, elsewhere \$231 \\ Patrick MacCarthy}

As analytical chemistry has evolved, it has focused primarily on the determination of substances within the controlled confines of a fixed laboratory. While there has always been an interest in conducting analyses where a sample is actually found, such onsite measurements have generally been found impractical for the more complex determinations that require highly sophisticated instrumentation and methodology.

Nevertheless, there has been a constant effort to adapt even the most sophisticated methods for portable use in the field. Such efforts introduce the inevitable challenges of instrument ruggedness, availability of appropriate power supplies, and control of operating parameters.

Field measurement is necessary when samples need to be analysed at their site of occurrence, as, for example, when dealing with unstable analytes; when immediate results are required, such as in detecting chemical or microbiological warfare agents on the battlefield; and when the analyst cannot be present, such as for analyses performed on the surfaces of other planets.

Field Analytical Chemistry and Techno$\log y$ is the first journal to focus on this area, and fills an important niche. It is international and interdisciplinary, publishing refereed, original research papers, short technical notes, reviews and so-called 'Outfield Reports'. The latter articles arise from what is described as "major field analytical projects involving multidisciplinary teams, often operating under highly demanding conditions". Stimulating editorials add to the journal's appeal.

Although the editorial board is international, with members representing nine different countries, and the journal policy is international in scope, 75 per cent of the articles across three issues came from US laboratories.

The journal has a professional, highquality appearance and is nicely laid out. The period from submission to acceptance is generally less than two months; the lag for actual publication also appears to be short, but is difficult to discern exactly as copies of the journal are identified by volume and issue number within a given year rather than by month of publication.

Patrick MacCarthy is in the Department of Chemistry and Geochemistry, Colorado School of Mines, Golden, CO 80401, USA.

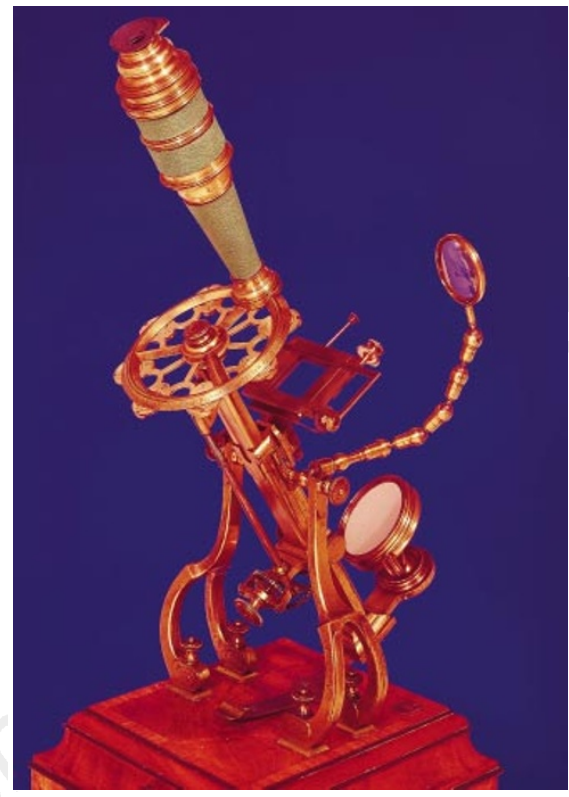

The Prince of Wales microscope, c. 1755.

\section{A site for sore eyes}

Microscopy and Microanalysis

Editor-in-chief Dale E. Johnson

Springer. 6/yr. \$539 (institutional)

\section{L.M.Brown}

Microscopes are to modern science what telescopes are to astronomy: a great enabling technology, which has brought about a considerable part of what is now undergraduate physics. Yet microscopy remains a discipline for professionals, and stands quite aloof from mainstream academic science, whether biological or physical.

Technical developments, often carrying in their train consequences of the utmost importance, such as new routes to the fabrication of devices on the nanometre scale, are announced either at specialized conferences or in the pages of Nature, but not usually in journals of physics or biology. So journals that cover conference programmes and details of trade exhibitions play a special role. They are sought after by every group that develops or applies microscopical techniques, especially when they concern structure and function of materials, whether biological or inorganic. The latest such publication is Microscopy and Microanalysis, the official journal of several major microscopical societies of North and South America. It is affiliated to the major European societies, and makes a fair bid to become the international house journal of microscopists.

It boasts an editorial board filled with eminent American pioneers of microscopy, nicely balanced between those concerned with biological problems and those concerned with the science of materials. North Americans predominate, but there is a smattering of Europeans and South Americans. 